\title{
SURFACE PLASMON RESONANCE IMMUNOSENSORS: SENSITIVITY CONSIDERATIONS
}

\author{
R.P.H. KOOYMAN*, H. KOLKMAN, J. VAN GENT and J. GREVE \\ Department of Applied Physics, University of Twente, P.O. Box 217, 7500 AE Enschede (The \\ Netherlands)
}

(Received 11th April 1988)

\section{SUMMARY}

Some aspects of improving surface plasmon resonance response in immunosensing applications are considered. Both from calculations and experiments, it was found that maximum sensitivity is obtained for a silver layer about $55 \mathrm{~nm}$ thick in direct contact with the species to be quantified. Application of an intermediate layer with high permittivity can be useful in suppressing background responses. Experimentally, a protein surface-coverage fraction of ca. 0.1 could be measured, corresponding to ca. $10^{-10} \mathrm{~mol} \mathrm{l}^{-1}$ antibody.

In recent years, the development of immuno-type biosensors has become a lively research topic [1-3], because of the potentially very high selectivity of these sensors. In an immunosensor, a "selector" molecule, the (mono- or polyclonal) antibody, is immobilized onto a surface. In principle, only the corresponding antigen will bind to the antibody. This immune reaction alters a physical parameter (e.g., charge or/and charge distribution, thickness of surface layer, etc.) which can be measured by some suitable method. In the subclass of optical biosensors, the exploitation of the concept of the evanescent wave [4] results in optical measuring systems that are highly selective to only surface parameters. This is particularly important in applications where the bulk composition of the measuring fluid is variable, such as in blood.

Among the various evanescent-wave methods, the detection of surface plasmon resonances (SPR) $[5,6]$ has the definite advantage that intrinsic properties of the immuno-complex are monitored, such as refractive index and thickness of the surface layer; in principle, this property allows the sensor to be applied in in vivo systems. The SPR experiment can be done by recording the reflectance as a function of the angle of incidence for light falling on a metal-coated glass surface. At some angle $\theta_{\mathrm{r}}$, a sharp minimum in the reflectance occurs, indicating the excitation of a surface plasmon (cf. Fig. 1a). This angle is strongly dependent on the dielectric profile $\epsilon(z)$ in the immediate vicinity of the metal layer. A more detailed description of the physics of SPR has been given by Raether [7]. 
In some previous reports $[5,6,8]$ the usefulness of SPR experiments for monitoring surface immune reactions was clearly demonstrated. Here, some factors are discussed which might contribute to maximization of the SPR-sensor response. Emphasis is given to a quantitative description of SPR in a protein system, from which conclusions can be drawn on dimensioning a practical SPR sensor. Some experiments on the human serum albumin (HSA) immune reaction are also described; they provide both information on the reliability of the SPR description and on the chemistry of the immune reaction.

\section{EXPERIMENTAL}

The SPR experiments were done with a slightly modified Kretschmann ATR configuration [9] (see Fig. 1b): p-polarized light of a 10-mW polarized 633$\mathrm{nm} \mathrm{He} / \mathrm{Ne}$ laser was directed onto a BK-7 prism placed on a rotation stage with $10^{-2}$ degree angular resolution. The reflectance $R=R(\theta)$ was monitored by a large-area photodiode (UDT PIN 44D); via an electronic divider (cf. Fig. $1 b$ ), its output was fed to a strip-chart recorder. This configuration resulted in a noise level equivalent to $\Delta R \approx 10^{-3}$. As indicated in Fig. $1(\mathrm{~b})$, the measuring solution (volume $\approx 2.5 \mathrm{ml}$ ) was brought into optical contact with the prism via the combination of a metal-coated microscope glass cover slip (diameter about $1.8 \mathrm{~cm}$; refractive index $n=1.53$ ) and a film of matching oil (refractive index $n=1.52$ ), thereby avoiding the delicate procedure of cleaning and coating the prisms between successive experiments.

Cover slips were coated with silver or gold by a vapour-deposition procedure, yielding a layer thickness of ca. $50 \mathrm{~nm}$. Gold layers proved to have the better adhesion properties. In some cases, the metal layers were spin-coated with a

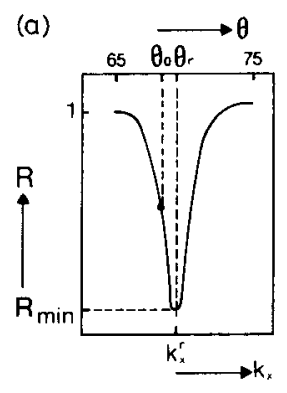

(b)

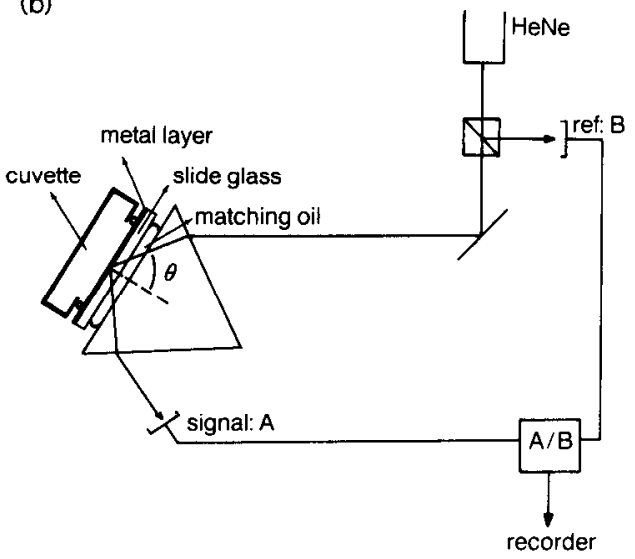

Fig. 1. Detection of surface plasmons: (a) the reflectance $R$ is recorded as a function of $\theta$; (b) the experimental set-up. 
polystyrene layer of about 20-nm thick. All thickness measurements were done with a surface profiler (Sloan Dektak).

Bovine serum albumin (BSA), HSA and polyclonal aHSA (Sigma) were checked for purity by gel electrophoresis; for HSA and BSA essentially one band was seen; for aHSA several bands were detected. In the present experiments, only the immuno-active aHSA molecules were expected to react selectively, so it was decided to determine the immuno-active fraction of aHSA molecules rather than to purify the aHSA samples.

Nominal concentrations of $2 \times 10^{-7}, 2 \times 10^{-8}$ and $2 \times 10^{-9} \mathrm{~mol} \mathrm{l}^{-1}$ aHSA were prepared in phosphate buffer, $\mathrm{pH}$ 7.1. The immunological activity of aHSA was found, by a fluorescence procedure [10], to be about $10 \%$, so that the effective concentrations ranged from $2 \times 10^{-8}$ to $2 \times 10^{-10} \mathrm{~mol} \mathrm{l}^{-1}$. HSA or aHSA was adsorbed to the metal layer by physical adsorption from a $10^{-5} \mathrm{~mol}$ $1^{-1}$ solution in phosphate buffer. The adsorption process was monitored by recording the reflectance in a SPR set-up as a function of time for some fixed angle $\theta$ [6]. After completion of the adsorption process, expectedly resulting in a monolayer surface coverage ( $\mathrm{cf}$. the section on the immune reaction), the protein-covered metal surface was rinsed and the cuvette solution was replaced by an aHSA or HSA solution of known concentration. The occurrence of an immune reaction resulted then in a further time-dependent change of the reflectance, which was recorded at an angle $\theta_{0}=\theta_{\mathrm{r}}-\Delta \theta$ corresponding to $R \approx 0.2$ (cf. Fig. 1a). This choice of $R$ kept the measurements approximately within the linear range of the angle-dependent reflectance.

\section{SENSITIVITY CONSIDERATIONS}

\section{Definition of sensitivity; calculation procedure}

The immune reaction is considered here as a time-dependent change of the permittivity profile $\epsilon(z)$ at the metal/water interface. This is illustrated in Fig. 2 , together with some typical values for $\epsilon$ and $z$. If $\epsilon_{\mathrm{p}_{1}}=\epsilon_{\mathrm{p} 2} \equiv \epsilon_{\mathrm{p}}$, such as is the case for the aHSA-HSA system [11], then the sensitivity can be defined as

$S_{\theta_{0}}=\left(\mathrm{d} R(\theta) / \mathrm{d} t_{\mathrm{p}}\right)_{\theta_{0}}=\left[\left(\mathrm{d} R / \mathrm{d} k_{x}\right)\left(\mathrm{d} k_{x} / \mathrm{d} t_{\mathrm{p}}\right)\right]_{\theta_{0}}$

where $R(\theta)$ is, as before, the reflectance at some specified angle $\theta, t_{\mathrm{p}}$ is the protein layer thickness, and $k_{x}$ is the component of the wavevector tangential to the layer system. On assuming that the lineshape of $R(\theta)$ is not dependent on $t_{\mathrm{p}}$, Eqn. 1 (a) can be rewritten as

$S_{\theta_{0}}=\left(\mathrm{d} R / \mathrm{d} k_{x}\right)_{\theta_{0}}\left(\mathrm{~d} k_{x}^{\mathrm{r}} / \mathrm{d} t_{\mathrm{p}}\right)$

where $k_{x}^{\mathrm{r}}$ is the component of the plasmon-resonant wavevector tangential to the layer system. The relation between $k_{x}$ and the angle $\theta$ in Fig. 1 is given by

$k_{x}=(2 \pi / \lambda) n_{\mathrm{g}} \sin \theta$ 


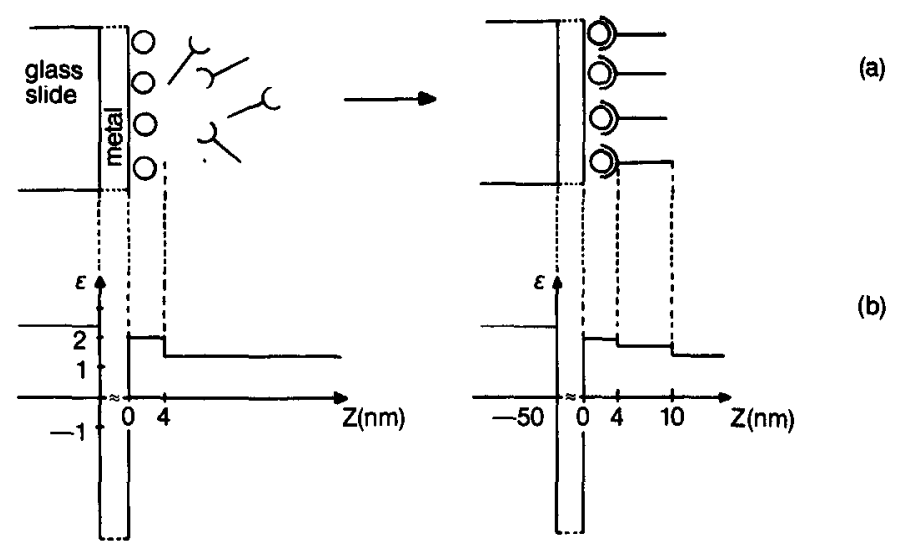

Fig. 2. The immune reaction (a) represented by a change of the permittivity profile (b). (0) antigens adsorbed to surface; $(Y)$ antibodies specifically binding to antigens. Each molecular layer corresponds to a certain thickness and permittivity; $\epsilon_{\text {silver }} \approx-16.0+j \times 0.5 ; \epsilon_{\text {protein }} \approx 2 ; \epsilon_{\text {water }} \approx 1.77$.

where $n_{\mathrm{g}}$ is the refractive index of the glass slide and $\lambda$ is the free space wavelength.

Thus, the factor $\mathrm{d} k_{x}^{\mathrm{r}} / \mathrm{d} t_{\mathrm{p}}$ describes the shift of the plasmon-dip, whereas $\mathrm{d} R$ / $\mathrm{d} k_{x}$ is a measure of the slope of the reflectance function $R=R(\theta)$, which is related to the plasmon resonance linewidth (see Fig. 1a). For a particular wavelength $\lambda$ and dielectric profile $\epsilon(z), k_{x}^{\mathbf{r}}$ can be determined by applying Maxwell's equations to a layer system such as that depicted in Fig. 2 (b). (Note that the two outermost layers, glass $\left(\epsilon_{0}\right)$ and water $\left(\epsilon_{4}\right)$, are semi-infinite.) To this end, a previously published 5 -layer dispersion equation [12] was used. The factor $\mathrm{d} R / \mathrm{d} k_{x}$ can be calculated by adapting the theory such as that developed by Raether [7] for a Lorentzian lineshape. In this theory, the plasmon linewidth is calculated on the assumption that two independent dissipating mechanisms are present: (1) the metal permittivity $\epsilon_{1}$ is a complex quantity, which results in electromagnetic energy absorption; (2) the non-radiative plasmons couple with ordinary light, which leads to radiation plasmon damping. Both effects can be accounted for in a complex wavevector $\mathbf{K}_{x}=k_{x}+j \gamma_{x}$ around the reflectance minimum. It can then be readily shown that, for a reflectance region around $R=0.5\left(1+R_{\min }\right)$, and for the case where the radiation plasmon damping is about the same as that caused by the imaginary part of $\epsilon_{1}$, the following relation holds:

$\mathrm{d} R / \mathrm{d} k_{x}=1 / 2 \gamma_{x}^{\mathrm{r}}$

where $\gamma_{x}^{\mathrm{r}}$ denotes the value of $\gamma_{x}$ at resonance. Equation 3 is strictly valid only for $\operatorname{Im}\left(\epsilon_{1}\right) \ll\left|\operatorname{Re}\left(\epsilon_{1}\right)\right|$. Additionally, although Eqn. 3 was derived for three layers, it is assumed here that it is valid, at least approximately, for up to five 
layers as well, in view of the relatively shallow dielectric profile for $z>0$ (see Fig. 2).

At resonance, the electric field, $E$, in each layer $i$ can be represented by

$E_{i}(x, z)=E_{0} \exp \left(j \mathbf{K}_{z_{i}}^{\mathrm{r}} z\right) \exp \left(j \mathbf{K}_{x}^{\mathrm{r}} x\right)$

where $\mathbf{K}_{z i}^{\mathbf{r}}$ is given by

$\mathbf{K}_{z_{i}}^{\mathrm{r}}=\left[\epsilon_{i}(2 \pi / \lambda)^{2}-\left(\mathbf{K}_{x}^{\mathrm{r}}\right)^{2}\right]^{1 / 2}$

as follows from the vector character of the wavevector $\mathbf{K}$. Thus, if $\mathbf{K}_{x}^{\mathbf{r}}$ can be calculated, the optical behaviour of the whole system is fully known: the electromagnetic field intensity distribution $|E|^{2}(z)$ and the penetration depth at resonance $1 / \operatorname{Im}\left(\mathbf{K}_{z_{2}}^{\mathrm{r}}\right)$ can then be calculated from Eqns. 4; both give valuable information on the surface probing properties of the plasmon field.

Now the problem of optimizing the SPR sensor system can be defined by formulating two requirements which ideally have to be met simultaneously (1) $S$ in Eqn. 1 should be a maximum, and (2) in order to minimize the response $I=\mathrm{d} R / \mathrm{d} \epsilon_{\mathrm{b}}$ to variations in the bulk properties of the measuring fluid, the evanescent field in the solution should be minimized, and the relative field intensity in the protein layer should be maximized. This latter requirement points to the use of an intermediate layer between metal and adsorbate with high permittivity, in view of the continuity of the dielectric displacement at each of the interfaces. Other important parameters are the choice of the metal coating and of its thickness.

\section{Results of calculations}

From Raether's theory [7], it was calculated that Eqn. 3 can be applied to silver layers with thicknesses around $55 \mathrm{~nm}$. For gold layers, the validity of Eqn. 3 is somewhat more questionable in view of its 633 -nm permittivity ratio: $\left|\operatorname{Im}\left(\epsilon_{1}\right) / \operatorname{Re}\left(\epsilon_{1}\right)\right| \approx 0.1$.

In Fig. 3 are shown some results obtained by varying the thickness of an intermediate layer $(\epsilon=2.53)$. It is clearly seen that the integrated intensity in the protein layer relative to that in the bulk phase is higher in the 5-layer system than in the 4-layer system. Simultaneously, some field intensity is stored in the intermediate layer, the proportion of which proves to increase strongly with its thickness (Fig. 3c). At the position of maximum field intensity in the protein layer relative to the total field present in the system, a maximum in $\mathrm{d} k_{x}^{\mathrm{r}} / \mathrm{d} t_{\mathrm{p}}$ is expected, which for this configuration turns out to be around a layer thickness of ca. $20 \mathrm{~nm}$ (Fig. 4b). Unfortunately, however, deposition of high index material also results in a larger SPR linewidth (see Fig. 4a). It must therefore be concluded that the use of a high-index intermediate layer will reduce the sensitivity $S$ (cf. Fig. 4c). Such a layer will, however, simultaneously reduce the sensitivity to bulk index changes as can be inferred from Fig. 3(c). From these model calculations, it emerges clearly that it is not possible to meet 

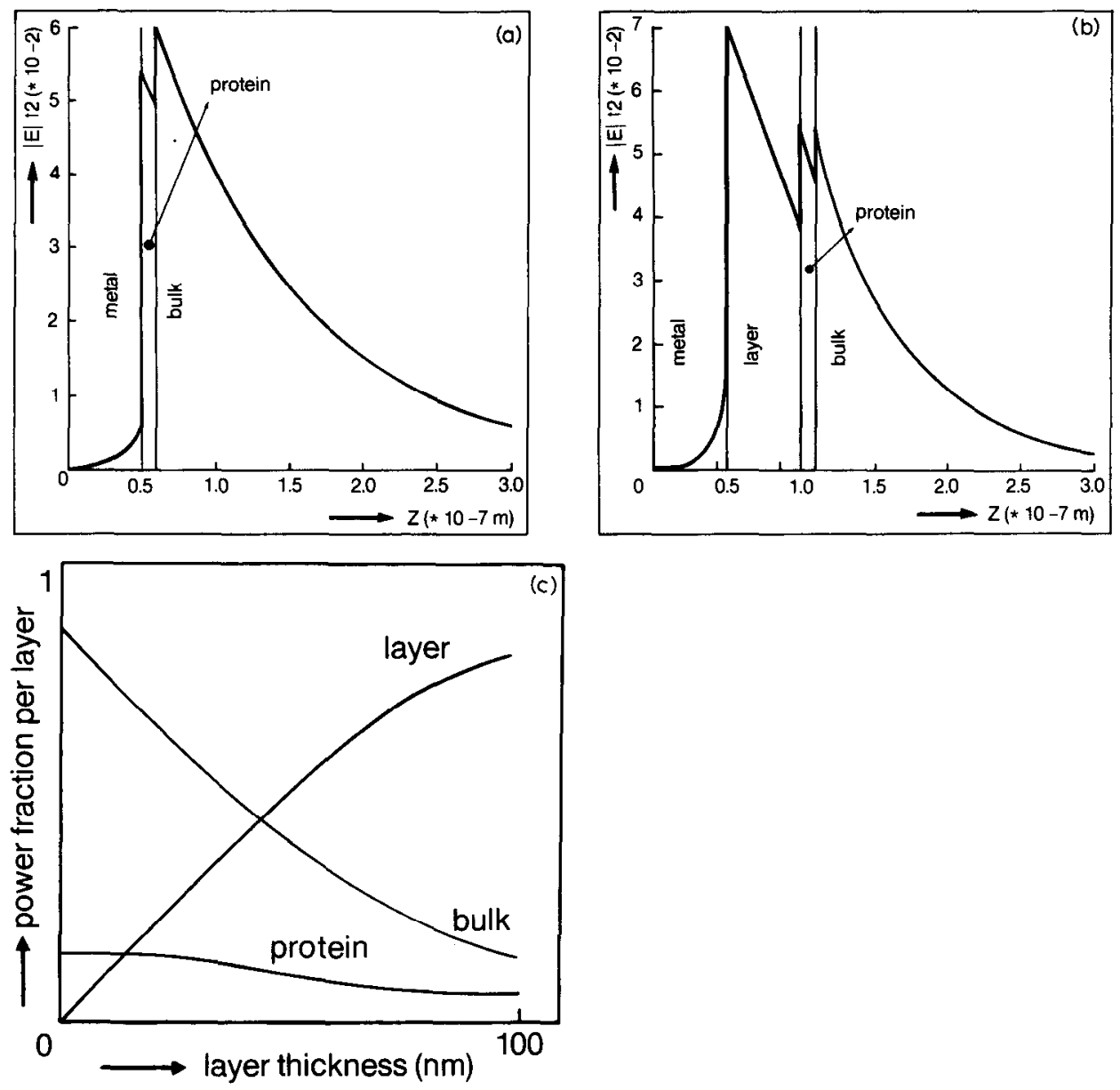

Fig. 3. Field intensity profiles in the absence (a) and presence (b) of an intermediate layer with $\epsilon=2.53$ and $d=20 \mathrm{~nm}$; integrated field intensities of (a) and (b) are kept constant. (c) The relative field intensities over the different layers as a function of the thickness of the intermediate layer.

simultaneously the two requirements mentioned in the previous section; rather, the configuration of a layer system in a SPR sensor should be selected with heavy bias given to the characteristics of the measuring solution. In this context, it is interesting to calculate the SPR response for different wavelengths $\lambda$ : from the known $\epsilon(\lambda)$ for silver [13], it was found that, although both the absolute sensitivity, $S$, and bulk interference, $I$, increase somewhat with increasing wavelength, $S / I$ decreases almost linearly with $\lambda$, mainly because of the large evanescent penetration depths at large $\lambda$.

Another point of interest is the choice of the metallic layer. For silver films around $50 \mathrm{~nm}$, plasmon dips with $R_{\min } \approx 0.04$ are found [14]. For the present 

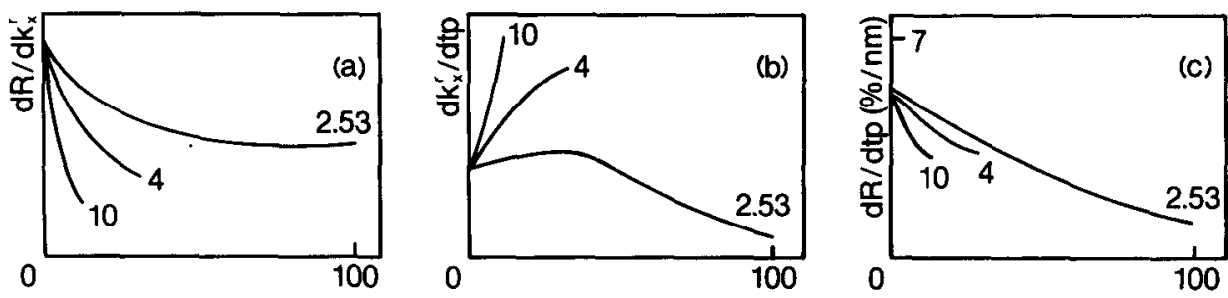

layer thickness $(\mathrm{nm})$

Fig. 4. The behaviour of the two factors (a,b) constituting the sensitivity $S(\mathrm{c})$ as defined in Eqn. 1. The parameter in the Figure is the permittivity of the intermediate layer.

dielectric profile, this value is calculated to shift to ca. $56 \mathrm{~nm}$; however, the stability of deposited silver layers is relatively poor when exposed to ambient atmosphere. As gold films are better in this respect, the SPR properties of such layers were calculated, although it can be predicted that these will be inferior to those of silver in view of the relatively high value of the imaginary part of the gold permittivity. The layer thickness at which a minimum reflectance occurs at the resonant wavevector was calculated to be around $40 \mathrm{~nm}$ for a gold/water or gold/protein interface. When, for this thickness, $S$ was calculated, the ratio found was $S_{\mathrm{Ag}} / S_{\mathrm{Au}} \approx 3$, which indeed proves to be a consequence of the increased linewidth of the gold SPR dip.

\section{EXPERIMENTAL RESULTS AND DISCUSSION}

Three types of surface reactions were studied: (I) HSA immobilized, aHSA in solution; (II) aHSA immobilized, HSA in solution; and (III) BSA immobilized, aHSA in solution.

\section{Comparison of theory and experiments}

In order to investigate the level of reliability of the above assumptions and calculations, several experiments were done on reaction I. First, it was found that at the start and end of the experiments, the half-reflectance linewidth remained constant (for a 53-nm silver layer, $\Delta \theta \approx 2.1^{\circ}$, measured outside the prism), from which it can be concluded that Eqn. 1(b) is applicable.

The following results were also obtained: (1) maximum sensitivity was found for silver layers at $53 \pm 2 \mathrm{~nm}$ (calculated, $56 \mathrm{~nm}$ ); (2) deposition of an intermediate $20-\mathrm{nm}$ polystyrene layer $(\epsilon=2.53)$ between silver and adsorbate resulted in a $20 \%$ reduction of sensitivity (calculated, $30 \%$ ); (3) the use of gold layers lowered the sensitivity by a factor of 4 compared to silver layers (calculated factor, 3 ). These findings indicate that the proposed calculations provide a reasonably accurate description. Discrepancies presumably stem from the circumstance that permittivities and effective layer thicknesses for the metal layer and the intermediate layer are not exactly known, as they may 
depend upon the deposition procedure [15] and/or the adjacent fluid layer [16]. Additionally, surface roughnes of the deposited layers and/or the protein adsorbate, which is not included in the calculations, may contribute to lower sensitivity.

\section{The immune reaction}

First, the thickness of the adsorbed HSA layer was evaluated. From the measured shift of the SPR angle $\left(\Delta \theta=0.2^{\circ}\right)$ after completion of the adsorption process, and the value of the HSA permittivity $(\epsilon \approx 2)[11]$, a layer thickness of $4 \mathrm{~nm}$ was calculated, in agreement with the dimensions of the HSA molecule. This can be interpreted as an indication of monolayer coverage of the metal layer; but it must be realized that only spatially averaged quantities were determined.

Next, the characteristics of reaction I were compared with those for reaction III. It was found that the reaction rate for the non-specific aHSA/BSA reaction was about half that for the aHSA/HSA reaction; and that the total reflectance change for the aHSA/BSA reaction was about half that for the aHSA/ HSA reaction. Apparently, the aHSA/BSA reaction is even slower than in the diffusion-limited reaction which is generally found in protein adsorption processes [17]. The second observation points to the circumstance that the equilibrium constant $K$ for the aHSA/BSA reaction is relatively small; if Langmuirtype adsorption behaviour is assumed, it can be estimated that $K_{\mathrm{BSA}} / K_{\mathrm{HSA}}<0.1$. Both these findings give further confidence in the interpretation of the aHSA/ HSA interaction as a pure immune reaction.

In another experiment, reaction II was monitored. Because of the smaller molecular dimensions of HSA (diameter $4 \mathrm{~nm}$ ) relative to those of aHSA (diameter $6 \mathrm{~nm}$ ), it would be expected for a non-specific aHSA/HSA reaction that the SPR shift caused by HSA coupling (reaction II) would be about $2 / 3$ of that caused by aHSA coupling (reaction $\mathbf{I}$ ). However, a reduction in the response by a factor of about 40 was found for identical molar quantities of aHSA and HSA. This is ascribed to the following aspects: in contrast to reaction I, the orientation of aHSA on the surface is important in reaction II if the observed aHSA/HSA reaction is indeed an immune reaction. If it is assumed that aHSA is randomly orientated on the surface, a rough estimate shows that at most about $25 \%$ of the antibodies have the proper orientation necessary for specific binding. Of that $25 \%$, only $10 \%$ is immuno-active (cf. experimental section). Taking into account the different dimensions of HSA and aHSA and the presence of two binding sites on aHSA, a net signal reduction by a factor of 30 would be expected in experiment II compared to that in experiment I. Although the factor calculated in this way seems to give a reasonable explanation of the experimentally found factor 40 , another conclusion is more important in the present context: apparently the contribution of non-specific aHSA/HSA interactions is fairly small; if it is assumed that the $25 \%$ well- 
orientated aHSA molecules in reaction II interact only non-specifically with HSA, it follows that less than $3 \%$ of the number of aHSA molecules participating in reaction $I$ is involved in a non-specific reaction.

Finally, the concentration dependence of reaction I was studied. Figure 5 depicts some typical results. It can be seen that $\Delta R$ for $2 \times 10^{-10} \mathrm{~mol} \mathrm{l}^{-1}$ aHSA is well above the noise level $\Delta R \approx 10^{-3}$. For different batches of metal-coated glass slides, the reproducibility of these results was fair: $\Delta[\Delta R(t=\infty)] \leqslant 0.1$; within one batch the results were reproducible within $\Delta R \approx 0.05$. This latter reproducibility could be reached only for relatively freshly prepared glass slides (age $\leqslant 3$ days). Apparently, the SPR response is highly dependent on the microstructure of the deposited layer and so on the detailed conditions of the preparation procedure, ageing of metal coatings may also have an appreciable effect. It is evident that these points need to be solved before the SPR concept can be introduced as a practical sensor system.

It was also noted that for each of the concentrations a plateau value was reached; the total reflectance change $\Delta R$ for the $2 \times 10^{-8} \mathrm{~mol} \mathrm{l}^{-1}$ solution was calculated to correspond to a total layer deposition of ca. $10 \mathrm{~nm}$, given a permittivity $\epsilon_{\mathrm{p}}=2$ [11] and given the fact that the present calculations showed that $\Delta R$ is proportional to $\Delta t_{\mathrm{p}}$ to a reasonable approximation. Although this thickness corresponds well to the estimated dimensions of the immuno-complex, it should be added that this number depends strongly on the choice of $\epsilon_{p}$. Clearly, separate measurements of $\epsilon_{p}$ and layer thickness would add useful information on the nature of the antigen/antibody interaction and could assist in improving the theoretical description of the SPR sensor system.

Another feature of Fig. 5 is that the plateau values differ for different analyte concentrations, contrary to expectations for an immune reaction. If the immune reaction is considered as a simple equilibrium reaction, then from the concentration dependence of the plateau values an equilibrium constant $K \approx 10^{9}$

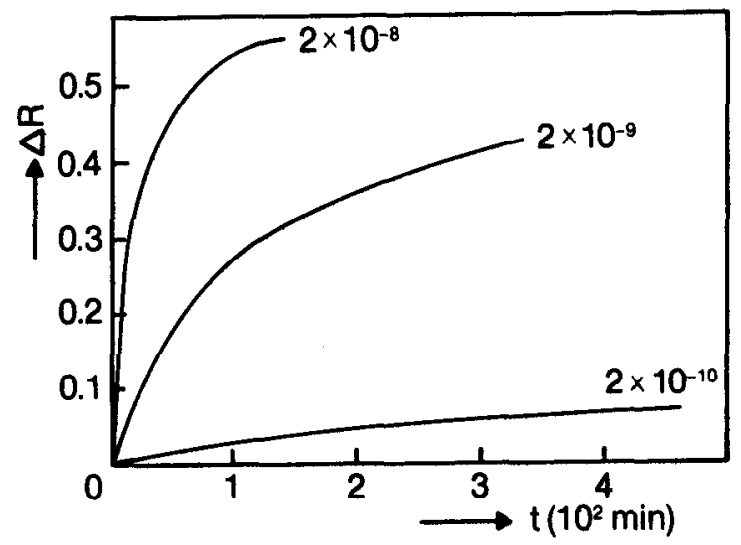

Fig. 5. Measured reflectance changes $\Delta R$ for different concentrations of aHSA ( $\left.\mathrm{mol} \mathrm{l}^{-1}\right)$. 
mol $\mathrm{l}^{-1}$ can be estimated. In the present sample cell, depletion certainly takes place, so that this number should be taken as a lower boundary value.

\section{Conclusions}

From this work, the following main points can be inferred. First, application of Maxwell's equations to the simplified situation as depicted in Fig. 2(b) appears to provide a reasonable description of SPR experiments in which protein deposition is monitored. Secondly, a proper choice of the layer system alone is not sufficient to improve the sensor sensitivity. A well-designed multiple-reflection SPR system along the lines previously described [18] could in principle reduce the loss of sensitivity associated with the increased SPR linewidth. However, this type of sensor presently has a much lower sensitivity than the ATR configuration used in this study. Thirdly, from the calculations outlined above, the choice of the layer system and the wavelength are expectedly important factors in reducing sensor responses that originate from variations in bulk refractive index or in temperature or in composition of the analyte solution. Nevertheless, in unfavourable cases, the use of an additional optical referencing system will certainly be necessary.

A sensitivity around $5 \times 10^{-2} / \mathrm{nm}$ was calculated for the SPR (cf. Fig. $4 \mathrm{c}$ ), whereas the experimental noise level was $\Delta R \approx 10^{-3}$. For an antibody with a diameter of ca. $5 \mathrm{~nm}$, this corresponds to a measurable average covered surface fraction of the order of $10^{-2}$. Below this region, the covered surface fraction and thus the reflectance is a very shallow function of the concentration, as is easily seen from the form of the Langmuir-type binding isotherms [17].

Thus, without a molecular amplifier system, such as that utilized in enzymelinked immunoassays, for example, it seems very difficult to detect concentrations below ca. $1 /(100 \mathrm{~K}) \mathrm{mol}^{-1}$ by methods which measure average surface concentrations. This number is in reasonable agreement with the above experimental results which indicate a sensitivity of better than $1 /(10 \mathrm{~K}) \mathrm{moll}^{-1}$.

Ms. Lian Keldermans, who also prepared the samples, is acknowledged for useful suggestions on determining the immunological activities. Dr. H.J.M. Kreuwel is acknowledged for helpful discussions on surface plasmons.

\section{REFERENCES}

1 C. Nylander, J. Phys. E, 18 (1985) 736.

2 J.F. Place, R.M. Sutherland and C. Dahne, Biosensors, 1 (1985) 321.

3 W.M. Reichert, C.J. Bruckner and J. Joseph, Thin Solid Films, 152 (1987) 345.

4 J.D. Andrade, R.A. Van Wagenen, D.E. Gregonis, K. Newby and J.N. Lin, IEEE Trans. Electron Devices, 32 (1985) 1175.

5 M.T. Flanagan and R.H. Pantell, Electron. Lett., 20 (1984) 969.

6 B. Liedberg, C. Nylander and I. Lundström, Sens. Actuat., 4 (1983) 299. 
7 H. Raether, in G. Hass, M.H. Francombe and R.W. Hoffman (Eds.), Physics of Thin Films, Vol. 9, Academic, New York, 1977, p. 145.

8 P.B. Daniels, J.K. Deacon, M.J. Eddowes and D.G. Pedley, Proc. 2nd Int. Meeting Chem. Sensors, Bordeaux, 1986, p. 534.

9 E. Kretschmann and H. Raether, Z. Naturforsch., Teil A, 23 (1968) 2135.

10 L. Keldermans and B.S.F. Altenburg, unpublished work.

11 P.A. Cuypers, Ph.D. Thesis, University of Limburg, Maastricht, The Netherlands, 1976.

12 C.A. Ward, K. Bhasin, R.J. Bell, R.W. Alexander and I. Tyler, J. Chem. Phys., 62 (1975) 1674.

13 American Institute of Physics Handbook, McGraw-Hill, New York, 1963.

14 E. Kretschmann, Z. Phys., 241 (1971) 313.

15 C.E. Reed, J. Giergiel, S. Ushioda and J.C. Hemminger, Phys. Rev., Part B, 31 (1985) 1873.

16 J.D. Pollard, G.W. Bradberry and J.R. Sambles, Solid State Commun., 63 (1987) 803.

17 J.D. Andrade, in J.D. Andrade (Ed.), Surface and Interfacial Aspects of Biomedical Polymers, Vol. 2, Plenum, New York, 1985.

18 H.J.M. Kreuwel, T.J.A. Popma, P.V. Lambeck and G.J. Gerritsma, Proc. 2nd Int. Meeting Chem. Sensors, Bordeaux, 1986, p. 692. 